\title{
In vitro and in vivo antimicrobial activity of water-soluble chitosan oligosaccharides against Vibrio vulnificus
}

\author{
BYUNG CHEOL LEE ${ }^{1}$, MYUN SOO KIM ${ }^{1}$, SANG HO CHOI $^{2}, \mathrm{KWANG}$ YOON KIM ${ }^{3}$ and TAE SUNG KIM ${ }^{1}$ \\ ${ }^{1}$ School of Life Sciences and Biotechnology, Korea University, Seoul 136-701; \\ ${ }^{2}$ National Research Laboratory of Molecular Microbiology and Toxicology, Department of \\ Agricultural Biotechnology, Seoul National University, Seoul 151-921; ${ }^{3}$ TBI Center, \\ Chonnam National University, Gwangju 500-757, Republic of Korea
}

Received April 9, 2009; Accepted June 10, 2009

DOI: 10.3892/ijmm_00000236

\begin{abstract}
Vibrio vulnificus is a Gram-negative bacterium that induces severely rapid pathological progress. In this study, we evaluated the antibacterial activity of two water-soluble chitosan oligosaccharides, COS A (MW, 10,000 Da) and COS B (MW, 1,000 Da), from 90-95\% deacetylated chitosan, against $V$. vulnificus in vitro and in vivo. Treatment with COS A resulted in significantly higher suppressive effects on the growth of $V$. vulnificus than treatment with COS B. The growth of $V$. vulnificus was inhibited within $1 \mathrm{~h}$ of treatment with water-soluble $\mathrm{COS} \mathrm{A}$ in concentrations ranging from 0.5 to $10 \mathrm{mg} / \mathrm{ml}$. Additionally, treatment with COS A completely inhibited $V$. vulnificus-induced cytotoxicity in human intestinal epithelial INT-407 cells, while COS B did not. Furthermore, the administration of COS A (0.1-0.5 mg per mouse) significantly increased the survival period of $V$. vulnificus-infected mice. The number of viable $V$. vulnificus in the spleen, liver, small intestine, and blood was significantly lower in COS A-treated mice than in untreated mice. Here, we clearly demonstrate that $\operatorname{COS} \mathrm{A}$ is a potential agent for the prevention and treatment of infection with $V$. vulnificus.
\end{abstract}

\section{Introduction}

Vibrio vulnificus is a Gram-negative estuarine bacterium that causes primary sepsis and gastrointestinal illness in humans. In addition, wound infection or oral infection via contaminated shellfish induces severe fulminant septicemia in susceptible individuals such as those with chronic liver disease (1). $V$. vulnificus induces a rapid disease process that results in extensive cellular damage. As a result, mortality exceeds $50 \%$ in septic patients, and increases to $>90 \%$ in patients who go

Correspondence to: Dr Tae Sung Kim, School of Life Sciences and Biotechnology, Korea University, Seoul 136-701, Korea E-mail: tskim@korea.ac.kr

Key words: Vibrio vulnificus, chitosan oligosaccharide, antimicrobial activity into shock shortly after admission to the hospital. The majority of fatal cases are caused by septic shock, which results from a variety of virulence factors of $V$. vulnificus. Putative virulence factors of $V$. vulnifcus, including capsular polysaccharide $(2,3)$, siderophores (4), hemolysin (5), matrix metalloproteinase, flagella (6), and RtxA toxin (7-9), have been reported in vivo and in vitro. These virulence factors may persistently activate the production of proinflammatory mediators such as tumor necrosis factor- $\alpha$, interleukin (IL)- $1 \beta$, IL-6, IL-8 and nitric oxide in the infected host $(10,11)$. Therefore, successful treatment of fulminant $V$. vulnificus infections requires highly active antimicrobial agents. In this study, we examined the anti-V. vulnificus activity of water-soluble chitosan oligosaccharides (COS) in vitro and in vivo.

Chitin and chitosan are abundant natural polysaccharides. Chitosan has attracted a great deal of interest due to its wide range of functions. Chitosan is a linear polysaccharide comprised of $\mathrm{N}$-acetyl-D-glucosamine and deacetylated glucosamine that shares characteristics with various glucoaminoglycan and hyaluronic acids; therefore, it is likely that chitosan also shares bioactivities associated with these compounds (12). Chitin and chitosan were shown to exert a stimulatory effect on macrophages, as well as chemoattractive effects on neutrophils in vitro and in vivo (13-16). Furthermore, recent advances provide insight into the health benefits of COS, which are partially hydrolyzed products of chitosan. COS are watersoluble due to their shorter chain lengths and free amino groups in D-glucosamine units. Specifically, many of these compounds were found to have beneficial biological effects including anti-tumor (17-19), antimicrobial (20-22), cholesterol-reducing (23-25), and free radical scavenging (26-28) activity. In addition to its lack of toxicity and allergenicity, the biodegradability and biocompatibility of chitosan and COS make it potentially useful for biomaterial, medical, and pharmaceutical applications (29). Several studies have demonstrated that the degree of deacetylation and molecular weight (MW) strongly influence the action of COS in biological systems $(22,29,30)$. In addition, the anti-microbial activity of COS was shown to be greatly dependent on polymerization or MW. Furthermore, the anti-microbial action of water-soluble chitosan and COS was shown to be dependent on the degree of deacetylation (29,31). 
Previously, we found that two types of water-soluble COS prepared from $90-95 \%$ deacetylated chitosan, COS A (MW, 10,000) and COS B (MW, 1,000), exhibited potential anti-inflammatory effects (32). In this study, we investigated the antibacterial activity of COS A and B against $V$. vulnificus as well as their inhibitory effects against $V$. vulnificus-induced cell cytotoxicity in vitro and in vivo.

\section{Materials and methods}

Animal cell culture and chemicals. The human intestinal epithelial INT-407 cell-line (ATCC CCL-6) was purchased from the American Type Culture Collection (ATCC, Manassas, VA, USA), and maintained at $37^{\circ} \mathrm{C}$ in $5 \% \mathrm{CO}_{2}$ in Minimum essential medium (MEM) supplemented with $10 \%$ fetal bovine serum (Gibco BRL, Grand Island, NY, USA) and antibiotics (10 unit/ml penicillin $\mathrm{G}$ and $10 \mu \mathrm{g} / \mathrm{ml}$ streptomycin) (growth medium). Water soluble COS A and B were provided by ECOBIO Inc. (Gwangju, Korea). COS A and B were 90-95\% deacetylated and their molecular weights were 10,000 and 1,000 Da, respectively. Eight-week-old female ICR mice (Samtaco Inc, Korea) were maintained and treated under standard conditions according to the animal care committee guidelines of Korea University (KUIACUC-1/5/2009-3).

Bacterial strains and growth conditions. The V. vulnificus strain MO6-24/O used in this study was isolated from patients $(7,8)$. The $V$. vulnificus bacteria were grown to log phase at $30^{\circ} \mathrm{C}$ in Luria-Bertani medium supplemented with $2 \% \mathrm{NaCl}$ LBS medium, after which they were diluted to $6 \times 10^{8} \mathrm{CFU} / \mathrm{ml}$ in LBS medium and then centrifuged and resuspended in antibiotic-free MEM prior to infection into INT-407 cells. The concentration of bacteria was confirmed via viable cell counts conducted on LBS agar.

In vitro broth cultures of $V$. vulnificus. The $V$. vulnificus inoculum size was $6 \times 10^{8} \mathrm{CFU} / \mathrm{ml}$. Variable amounts of COS $(0.5,1,2.5,5,10 \mathrm{mg} / \mathrm{ml})$ were solubilized in $20 \mathrm{ml}$ of growth medium ( $2 \% \mathrm{NaCl} \mathrm{LBS})$ and then tested for their ability to alter bacterial growth by spectrometry $\left(\mathrm{OD}_{600}\right)$. The $V$. vulnificus cultures were then incubated with aeration (150 rpm, gyratory shaker) for $5 \mathrm{~h}$ at $37^{\circ} \mathrm{C}$.

Infection protocol. Human intestinal epithelial INT-407 cells were infected with $V$. vulnificus as previously described $(7,8)$. Briefly, INT-407 cells were grown in the growth medium at $37^{\circ} \mathrm{C}$ in a $5 \% \mathrm{CO}_{2}$ incubator. Next, cells were seeded onto 6$\left(8 \times 10^{5}\right.$ cells/well $)$ and 96 -well $\left(2 \times 10^{4}\right.$ cells/well) culture plates and then cultured for $24 \mathrm{~h}$ in antibiotic-free growth medium. Prior to infection, the bacteria were centrifuged for $3 \mathrm{~min}$ at 5,000 rpm, resuspended, and adjusted to $6 \times 10^{8} \mathrm{CFU} / \mathrm{ml}$ in antibiotic-free MEM medium. The bacterial suspensions were then added to COS-treated or untreated-intestinal epithelial cells at various multiplicities of infection (MOI; the ratio of bacteria number to the number of epithelial cells), after which the infected cells were incubated for 1-4 $\mathrm{h}$ in antibiotic-free growth medium at $37^{\circ} \mathrm{C}$ in $5 \% \mathrm{CO}_{2}$.

Cytotoxicity assay. The bacteria-infected INT-407 cell cultures were aliquoted into a 96-well tissue culture plate (Nunc,
Roskilde, Denmark) as previously described $(7,8)$. The cytotoxicity was then determined by measuring the activity of lactate hydrogenase (LDH) in the supernatant using a cytotoxicity detection kit (Roche, Mannheim, Germany). The cytotoxic level was expressed as a percentage relative to the total LDH activity of cells that were completely lysed by $1 \%$ Triton X-100 $(7,8)$.

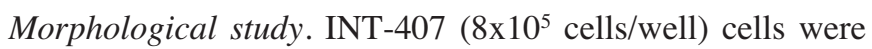
incubated with bacteria in a 6-well plate for $3 \mathrm{~h}$ at MOI 10 , after which the cells were washed with PBS. The cells were then fixed with $4 \%$ paraformaldehyde (Sigma) for $10 \mathrm{~min}$ at room temperature, washed and completely dried. Next, the cells were stained with Giemsa solution (Molecular Probe) for $1 \mathrm{~h}$ at room temperature. The cells were then washed twice with distilled water and dried, after which images of the specimens were acquired using a microscope (Olympus IX 71, Japan).

Survival of $V$. vulnificus-infected mice. Eight-week-old female ICR mice (Samtaco Inc, Korea) housed under specificpathogen free conditions were used for all experiments. Experiments reported in this study were performed using protocols approved by the Korea University Institutional Animal Care and Use Committee. The mice were intraperitoneally infected with $0.1 \mathrm{ml}$ of $250 \mu \mathrm{g}$ iron dextran (Sigma Aldrich, St. Louis, MO, USA) $30 \mathrm{~min}$ prior to injection with $V$. vulnificus. Next, mice were intraperitoneally injected with $1 \times 10^{3} \mathrm{CFU} / 0.1 \mathrm{ml}$ V. vulnificus. The mice were administered $0.2 \mathrm{ml}$ of chitosan oligosaccharide A solution $(0.1,0.2,0.5 \mathrm{mg}$ per mouse $)$ or a PBS control intraperitoneally, after which their survival status was assessed once an hour for $24 \mathrm{~h}$.

Quantitative analysis of bacteria in tissues. The $V$. vulnificusinoculated mice were sacrificed $7 \mathrm{~h}$ after infection, and the spleen, liver, blood, and small intestine lesions were then aseptically removed. The removed specimens were then homogenized in $2 \mathrm{ml}$ PBS using glass tissue homogenizers, after which the homogenates were diluted and plated on $2 \%$ $\mathrm{NaCl} \mathrm{HI}$ agar. The samples were then incubated at $37^{\circ} \mathrm{C}$ for $12 \mathrm{~h}$. In addition, blood $(50 \mu \mathrm{l})$ was collected from the tail vein of the mice and plated on $2 \% \mathrm{NaCl} \mathrm{HI}$ agar plates to determine the number of bacterial colonies.

Statistical analysis. A Student's t-test and one-way analysis of variance (ANOVA) followed by the Bonferroni method were employed to identify statistical differences between the values of the various experimental and control groups. P-values $<0.05$ were considered of statistical significance.

\section{Results}

$C O S$ A strongly inhibits $V$. vulnificus growth in vitro. Two types of water-soluble COS, COS A (MW, 10,000 Da) and COS B (MW, 1,000 Da), were prepared from 90-95\% deacetylated chitosan, after which their antibacterial activities against $V$. vulnificus were evaluated in vitro. As shown in Fig. 1A, the bacterial numbers of $V$. vulnificus MO6-24/O increased rapidly as incubation time increased. However, treatment with COS A at concentrations of $1,2.5,5$, and $10 \mathrm{mg} / \mathrm{ml}$ completely inhibited the growth of $V$. vulnificus throughout the incubation 
$\mathbf{A}$

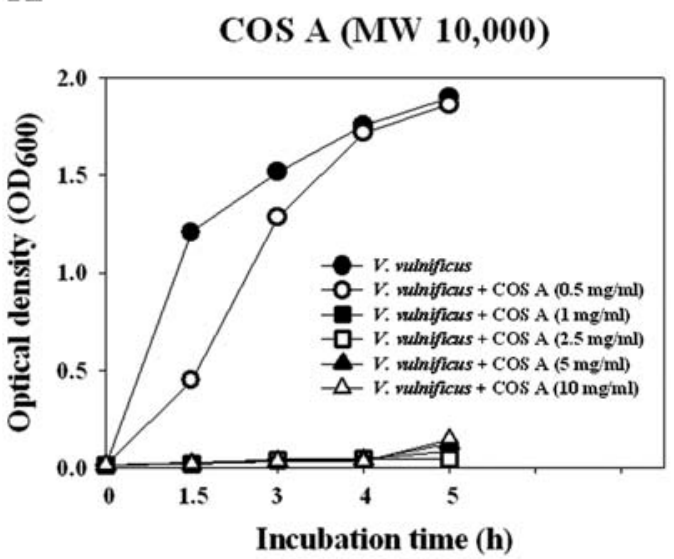

B

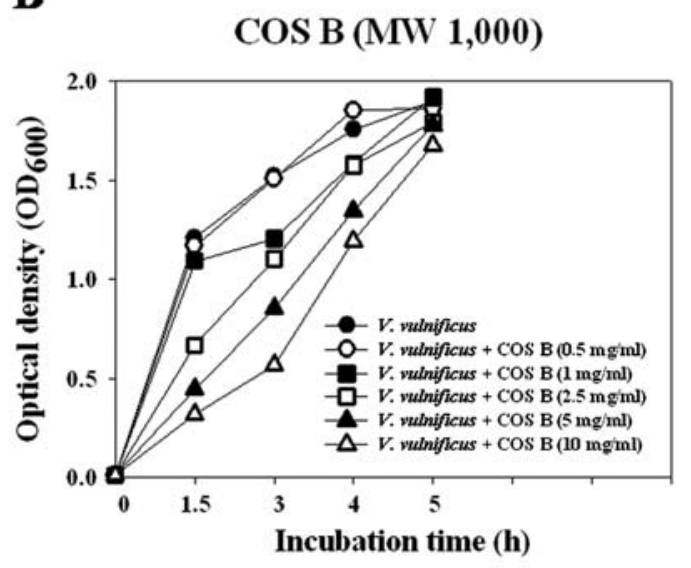

Figure 1. Inhibitory effects of the water-soluble chitosan oligosaccharides, COS A and B, on the growth of V. vulnificus. V. vulnificus was cultured with various concentrations of $\operatorname{COS} \mathrm{A}(\mathrm{A})$ and $\mathrm{COS} \mathrm{B}(\mathrm{B})$ at $37^{\circ} \mathrm{C}$ in $2 \% \mathrm{NaCl} \mathrm{LB}$ medium, after which the bacterial growth was evaluated by measuring the $\mathrm{OD}_{600}$.

period (0-5 h). In contrast, treatment with COS B slightly inhibited the growth of $V$. vulnificus for 1-4 h, even when it was administered at a concentration of $10 \mathrm{mg} / \mathrm{ml}$. After $5 \mathrm{~h}$ of incubation, the COS A no longer inhibited the growth of bacteria, regardless of the concentration. These findings indicate that COS with an MW of 10,000 Da (COS A) completely inhibits the growth of $V$. vulnificus, while COS with an MW of $1,000 \mathrm{Da}$ (COS B) does not inhibit the growth of $V$. vulnificus.

COS A inhibits the cytotoxicity of $V$. vulnificus against human intestinal epithelial cells. The cytotoxicity inflicted by a pathogen dictates its virulence. To determine if chitosan oligosaccharides exhibit an inhibitory effect against $V$. vulnificusinduced cytotoxicity, INT-407 cells were infected with $V$. vulnificus at different MOIs and incubation times in the absence or presence of COS A or B, after which the LDH levels released from the damaged cells were determined. As shown in Fig. 2A, the highest level of cytotoxicity was observed in INT-407 cells that were exposed to $V$. vulnificus at MOI 10 or 50 . However, treatment with COS A significantly inhibited $V$. vulnificus-induced cytotoxicity of INT-407 cells in a concentration-dependent manner. Indeed, for cells exposed to $V$. vulnificus at MOI 10 or 50 and were treated with $5 \mathrm{mg} / \mathrm{ml}$ $\operatorname{COS~A,~the~levels~of~LDH~activity~from~the~} V$. vulnificusinfected INT-407 cells were $\sim 3$-fold lower than the levels of the $V$. vulnificus-infected cells that were not treated with COS A. Furthermore, the inhibitory activity of COS A against $V$. vulnificus-induced cytotoxicity in INT-407 cells was observed throughout the entire treatment period, although the level of inhibitory activity differed as a function of treatment time (Fig. 2C).

Conversely, COS B exerted significantly lower inhibitory activity against $V$. vulnificus-induced cytotoxicity than COS A (Fig. 2B). Specifically, $22.67 \pm 1.51 \%$ of the LDH was released from intestinal epithelial cells that were infected with $V$. vulnificus at MOI 10 and then treated with $\operatorname{COS~A~for~} 3 \mathrm{~h}$, whereas $67.26 \pm 1.99 \%$ of the LDH was released from $V$. vulnificus-infected cells (MOI 10) that were treated with COS B for $3 \mathrm{~h}$ (Fig. 2).

Microscopic analyses were conducted as previously described to confirm the inhibitory effects of COS A on the cytotoxicity of $V$. vulnificus in INT-407 cells (8). Specifically, the size, regularity of the cell margin, and the morphological characteristics of the nuclei in the stained cells were assessed. As shown in Fig. 3A, marked cellular damage was observed in a large number of Giemsa-stained INT-407 cells that were infected with $V$. vulnificus, regardless of treatment with COS B. Furthermore, cytoplasmic loss and cellular damage, which are typical phenotypes of cell death, were observed in intestinal epithelial cells that were infected with $V$. vulnificus. Conversely, many healthy cells with intact cellular shapes were observed in V. vulnificus-infected cells treated with COS A. In addition, these cells did not exhibit damaged surfaces or cytoplasmic loss, which suggests that COS A protects the host cell against V. vulnificus-induced cytotoxicity (Fig. 3A).

To further examine COS A for inhibitory effects on the growth of $V$. vulnificus, all bacteria were isolated from untreated and treated groups at each incubation time point and plated on agar plates to determine the total numbers of bacterial colonies in each group. As shown in Fig. 3B, the number of bacteria increased sharply as the incubation time increased for epithelial cells infected with $V$. vulnificus in the absence or presence of COS B $(5 \mathrm{mg} / \mathrm{ml})$. However, the presence of COS A $(5 \mathrm{mg} / \mathrm{ml})$ in the cultures inhibited the growth of $V$. vulnificus throughout the incubation period. Specifically, the number of $V$. vulnificus was $(112.5 \pm 6.36) \times 10^{5}$ and $(99.6 \pm 3.54) \times 10^{5} \mathrm{CFU}$ for the untreated and COS B-treated cell cultures at $4 \mathrm{~h}$ post incubation, respectively. Conversely, the number of $V$. vulnificus was $(3.95 \pm 0.21) \times 10^{5} \mathrm{CFU}$ in COS A-treated cell cultures (Fig. 3B). Taken together, these results indicate that COS A significantly inhibits $V$. vulnificusinduced cell cytotoxicity through inhibition of the growth of V. vulnificus.

COS A administration prolongs the survival period of V. vulnificusinfected mice. Mice were infected with $V$. vulnificus and then administered COS A (0.5 mg per mouse) to determine if it increased the survival period. As shown in Fig. 4A, intraperitoneal inoculation with $1 \times 10^{3} \mathrm{CFU}$ of $V$. vulnificus resulted in the death of all mice within $10 \mathrm{~h}$. However, treatment with COS A significantly increased the survival of the V. vulnificus- 
A

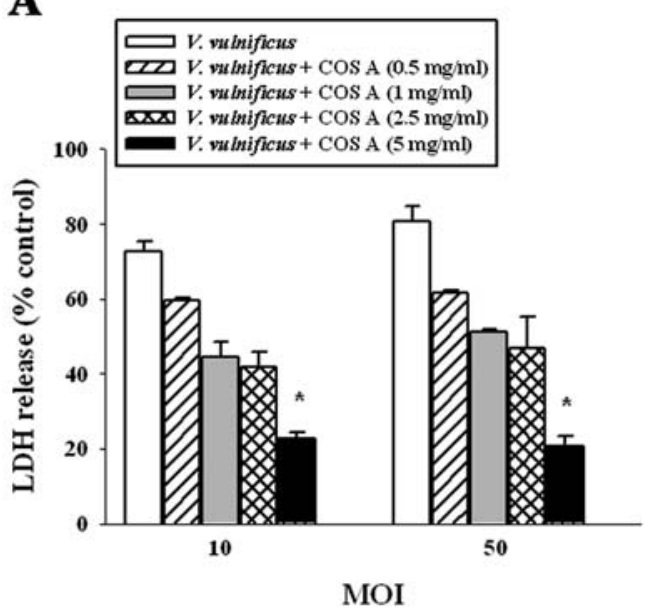

C

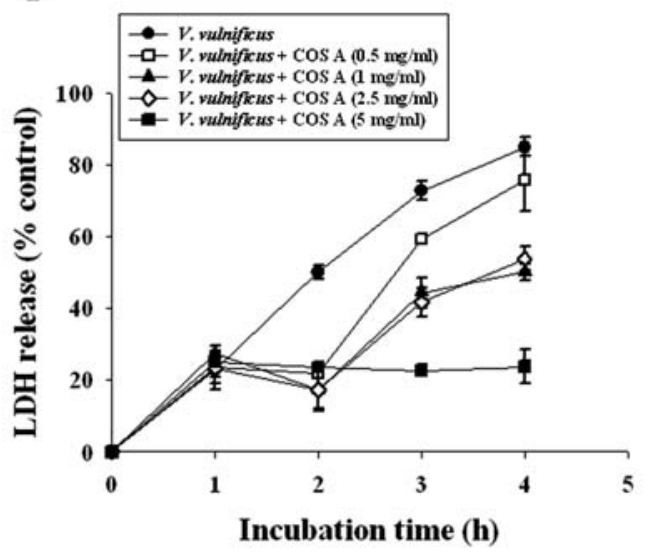

B

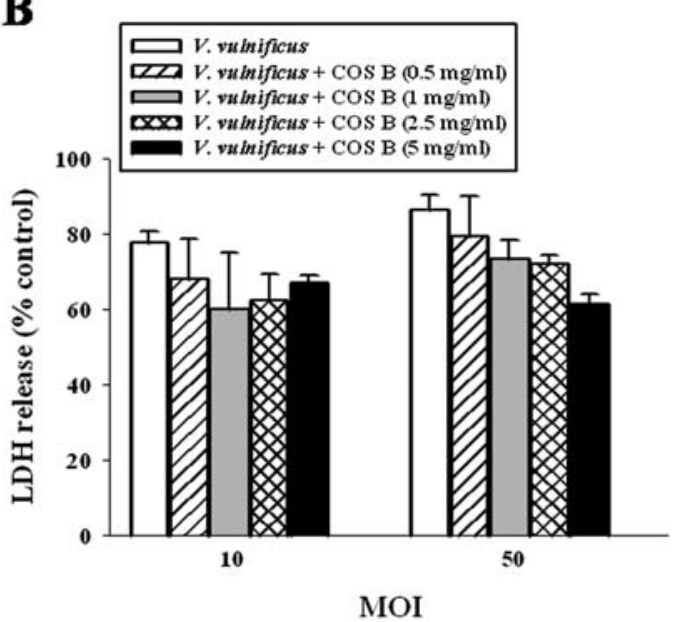

D

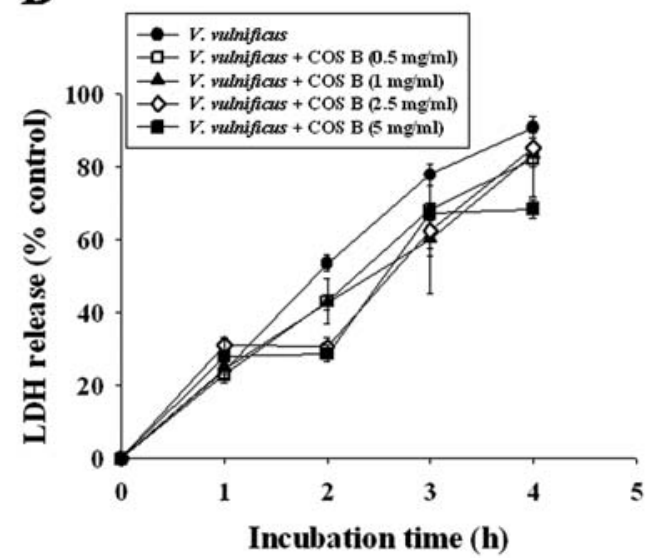

Figure 2. Suppression of the V. vulnificus-induced cytotoxicity in human intestinal epithelial cells by COS A and B. (A,B) INT-407 cells (2x10 4 cells/well) were infected with $V$. vulnificus for $3 \mathrm{~h}$ at MOI 10 and 50 in the absence or presence of COS A or B in a 96-well plate. (C,D) INT-407 cells (2x10 4 cells/well) were infected with $V$. vulnificus for 1-4 h at MOI 10 in the absence or presence of COS A or B in a 96-well plate. The cell cytotoxicity was then determined by an LDH release assay, as described in Materials and methods section. The data shown represent the means \pm standard errors ( $\mathrm{n}=3$ ). ${ }^{*} \mathrm{P}<0.05$, relative to a group infected with $V$. vulnificus in the absence of COS A.

infected mice. Indeed, 3 of 5 mice were alive for $24 \mathrm{~h}$ when the V. vulnificus-infected mice were treated with $\operatorname{COS} \mathrm{A}(0.1$, 0.2 , or $0.5 \mathrm{mg}$ per mouse).

To evaluate the role that COS A plays in the growth of $V$. vulnificus in vivo, we evaluated the post inoculation recovery of $V$. vulnificus isolated from various tissue samples obtained from treated and untreated mice. To accomplish this, mice were injected with $1 \times 10^{3} \mathrm{CFU} V$. vulnificus intraperitoneally, after which they were treated with $\operatorname{COS} \mathrm{A}(0.25 \mathrm{mg}$ per mouse). The spleen, liver, small intestine and blood were then removed from the mice at $7 \mathrm{~h}$ post infection, after which the number of $V$. vulnificus in each type of tissue was determined.

As shown in Fig. 4B, the numbers of bacterial colonies were significantly lower in all tissue types collected from mice that had been treated with COS A. Specifically, the number of $V$. vulnificus colonies recovered from the spleen, liver, and small intestine of the untreated mice was $(2.9 \pm 3.6) \times 10^{9}$, (5.4 \pm 7.1$) \times 10^{9}$, and $(2.56 \pm 12.9) \times 10^{10} \mathrm{CFU}$, respectively, whereas the number of colonies recovered from COS A treated mice was $(1 \pm 0.6) \times 10^{8},(3 \pm 1.2) \times 10^{8}$, and $(4 \pm 0.6) \times 10^{8} \mathrm{CFU}$, respectively. In addition, the blood collected from the untreated and COS Atreated mice contained $(297 \pm 12.0),(24 \pm 4)$, and $(1 \pm 1) \mathrm{CFU}$
$V$. vulnificus, respectively. Furthermore, the necropsy of $V$. vulnificus-infected mice at $7 \mathrm{~h}$ post infection revealed that there was edema, hemorrhage, vasodilation, and necrosis in the intestine, liver, and spleen samples collected from the untreated mice, but none of these symptoms were observed in samples collected from the COS A-treated mice. Taken together, these results suggest that COS A significantly inhibits the growth of $V$. vulnificus and $V$. vulnificus-induced illness in vitro and in vivo.

\section{Discussion}

Vibrio vulnificus is a Gram-negative bacterium that causes septicemia in humans who suffer from liver cirrhosis, haemochromatosis, immunocompromised conditions or diabetes $(33,34)$. V. vulnificus infection rapidly induces extensive cellular damage, which ultimately results in mortality of $>50 \%$ of septic patients. Therefore, highly antimicrobial agents are required for successful treatment of $V$. vulnificus infections. Chitosan oligosaccharides (COS) are produced chemically through acid and alkaline hydrolysis, physically through the use of high temperatures or enzymatically with chitinase, lysozyme, cellulase, lipase, or pronase $(35,36)$. The antimicrobial 
$\mathbf{A}$

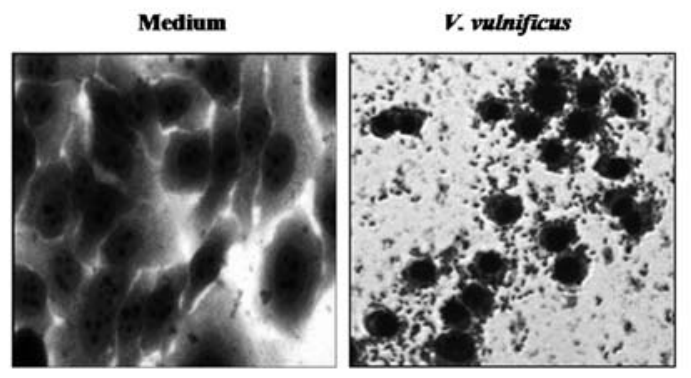

V. vulnificus $+\cos A(5 \mathrm{mg} / \mathrm{ml})$ V. vulnificus $+\operatorname{Cos} B(5 \mathrm{mg} / \mathrm{mI})$

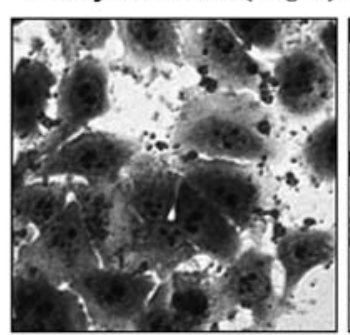

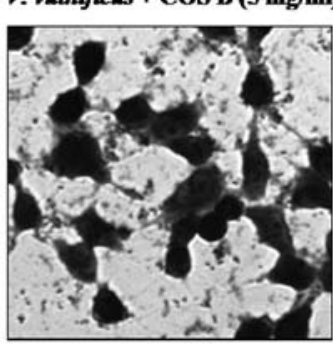

B

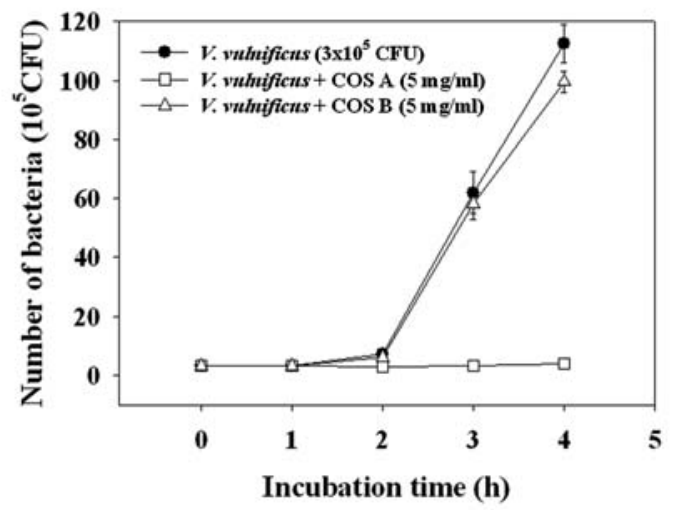

Figure 3. Inhibitory effects of COS A on the growth of $V$. vulnificus in human intestinal epithelial cells. (A) INT-407 (8x $10^{5}$ cells/well) cells were incubated with $V$. vulnificus for $3 \mathrm{~h}$ at MOI 10 in the absence or presence of $\operatorname{COS~A~or~B~}(5 \mathrm{mg} / \mathrm{ml})$ in 6-well plates. The culture plates were then centrifuged and washed twice with pre-warmed PBS (pH 7.4), after which they were fixed with 4\% paraformaldehyde. Next, the cells were washed twice with PBS, and then stained with Giemsa solution. The images of the specimens were then acquired using a microscope (Olympus IX 71, Japan). (B) INT-407 (3x104 cells/well) cells were incubated with $V$. vulnificus for 1-4 $\mathrm{h}$ at MOI 10 in the absence or presence of COS A or B $(5 \mathrm{mg} / \mathrm{ml})$ in 6-well plates. Both bacteria and INT-407 cells were then harvested and washed twice with pre-warmed PBS ( $\mathrm{pH}$ 7.4), after which they were lysed with $1 \%$ Triton X-100. Aliquots of the cell lysate were then plated on $2 \% \mathrm{NaCl} \mathrm{LB}$ agar after adequate dilution and the numbers of the bacterial colonies were then recorded.

A

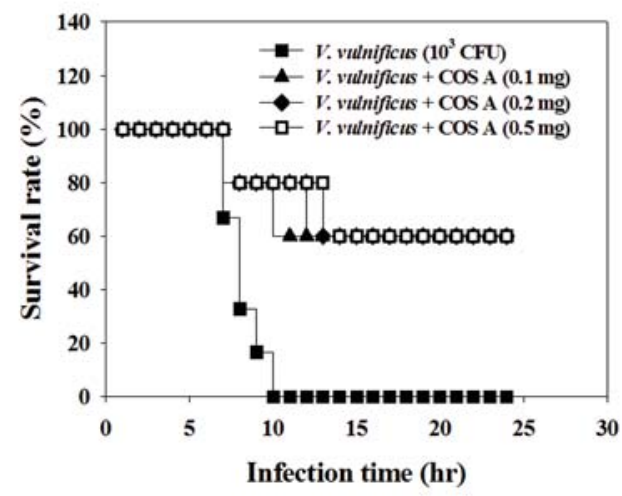

B
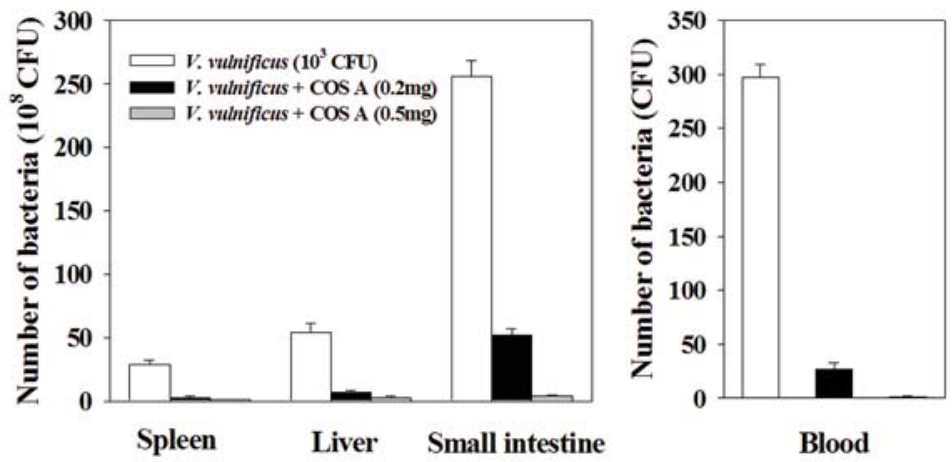

C

No treatment

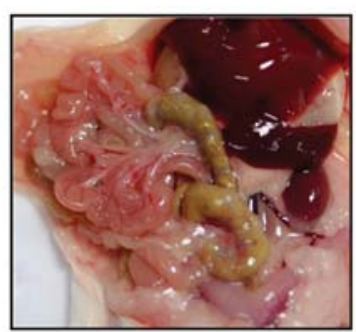

$\operatorname{COSA}(0.5 \mathrm{mg})$

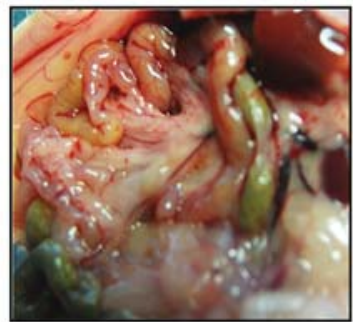

V. vulnificus $\left(10^{3} \mathrm{CFU}\right)$ $+\operatorname{Cos} A(0.5 \mathrm{mg})$

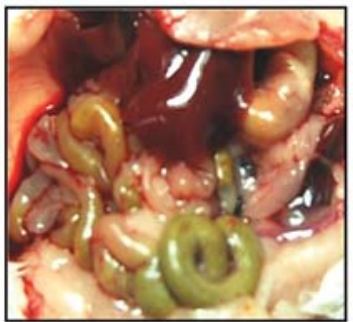

V. vulnificus (10 $0^{3}$ CFU)

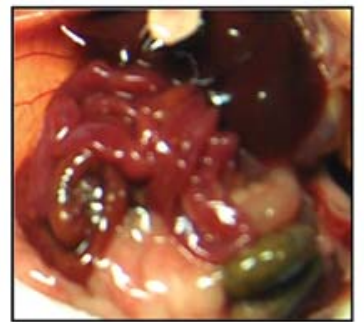

Figure 4. Prolongation of the survival of $V$. vulnificus-inoculated mice in response to COS A treatment. (A) Survival of $V$. vulnificus-inoculated mice. ICR female mice ( $\mathrm{n}=5$ per group) were intraperitoneally inoculated with $10^{3} \mathrm{CFU}$. vulnificus, after which they were treated with COS A ( $0.5 \mathrm{mg}$ per mouse). The survival of the mice was then recorded every hour for $24 \mathrm{~h}$. (B) Determination of the numbers of $V$. vulnificus colonies recovered from various tissues. ICR female mice ( $\mathrm{n}=5$ per group) were intraperitoneally inoculated with $10^{3} \mathrm{CFU}$ V. vulnificus, and then either treated with COS A ( $0.5 \mathrm{mg}$ per mouse), or left untreated. At $7 \mathrm{~h}$ after $V$. vulnificus infection, the spleen, liver, small intestine, and blood of $V$. vulnificus-infected mice were obtained. The specimens were then homogenized, diluted, and plated on $2 \% \mathrm{NaCl} \mathrm{HI}$ agar plates and incubated at $37^{\circ} \mathrm{C}$ for $12 \mathrm{~h}$, after which the number of bacterial colonies was counted. (C) The opened abdomen of V. vulnificus-infected mice with or without COS A treatment, as observed by a digital camera (Nikon D60). 
activity of COS is dependent on the COS and the microorganism $(21,24,26)$. The results of the present study demonstrate that water-soluble COS A has strong anti- $V$. vulnificus activity in intestinal epithelial cells and mice. Several studies have shown the degree that deacetylation and molecular weight influence the antimicrobial activity of COS $(29,30)$. COS A and B in this study were both $\sim 95 \%$ deacetylated.

The growth of $V$. vulnificus was more effectively suppressed by treatment with $10,000-\mathrm{Da} \operatorname{COS}$ A than with $1,000-\mathrm{Da}$ COS B. Indeed, the growth of $V$. vulnificus was completely inhibited throughout the entire incubation period by COS A at concentrations of $1-10 \mathrm{mg} / \mathrm{ml}$, while COS B had only weak inhibitory activity against $V$. vulnificus growth and did not have any inhibitory effect until after $5 \mathrm{~h}$ of treatment (Fig. 1). Furthermore, V. vulnificus-infected intestinal epithelial cells treated with COS A exhibited $60 \%$ less cytotoxicity than those treated with $\operatorname{COS} \mathrm{B}$, which indicates that $\mathrm{COS}$ A protected the intestinal epithelial cells from $V$. vulnificus-induced cell death. Moreover, no cytoplasmic loss or cellular damage was observed in intestinal epithelial cells that were infected with $V$. vulnificus in the presence of COS A. Additionally, treatment of $V$. vulnificus infected mice with COS A resulted in an increased survival rate when compared to the untreated and COS B-treated groups (Fig. 4A). Finally, the number of $V$. vulnificus colonies recovered from the spleen, liver, small intestine, and blood of COS A-treated mice was significantly lower than the concentration recovered from COS B-treated mice.

The reason for greater antibacterial activity against $V$. vulnificus observed in response to treatment with $10,000-\mathrm{Da}$ COS A when compared to treatment with 1,000-Da COS B remains unclear. Recent studies show that chitosan malate inhibits exotoxin production in S. aureus and A streptococci, and that weakens virulence factors such as secreted aspartyl proteinases (SAPs) (20). In addition, V. vulnificus has been shown to exert cytotoxicity toward Jurkat cells of T-lymphocytes through production of intracellular reactive oxygen species (ROS) (37). ROS generation is essential for both cell signaling and bacterial defense in innate immunity. However, excessive generation of ROS in host cells that are infected with bacteria is known to induce cell death. Recently, COS was found to have several beneficial biological effects including free radical scavenging activity (26-28), a stimulatory effect on macrophages, and chemoattractive effects on neutrophils in vitro and in vivo (13-16). Therefore, COS A may have a greater effect than COS B via the inhibition of cytotoxic factors such as cytolysin, metalloproteinase, elastase, and RTX toxin in $V$. vulnificus or through the suppression of excessive ROS by $V$. vulnificus-infected cells. However, further studies are needed to determine the mechanistic reasons for the differential antibacterial efficacy of COS A and B against V. vulnificus infection, as well as their therapeutic pathway and optimum treatment conditions.

Taken together, the results of this study clearly demonstrate that water-soluble COS A has strong antibacterial activity against $V$. vulnificus in vitro and in vivo and that the efficacy of COS was dependent on their molecular weight. These findings also suggest that the COS A (MW; 10,000) can be applied as a therapeutic agent for the treatment of $V$. vulnificus infectious disease.

\section{Acknowledgements}

This study was supported by a grant from the Marine Biotechnology Program funded by the Ministry of Land, Transport and Maritime Affairs (0552-200700001), and also by a grant of the Korea Healthcare Technology R\&D Project, Ministry of Health \& Welfare, Republic of Korea (A080777).

\section{References}

1. Ikeda T, Kanehara S, Ohtani T and Furukawa F: Endotoxin shock due to Vibrio vulnificus infection. Eur J Dermatol 16: 423-427, 2006.

2. Powell JL, Wright AC, Wasserman SS, Hone DM and Morris JG Jr: Release of tumor necrosis factor alpha in response to Vibrio vulnificus capsular polysaccharide in vivo and in vitro models. Infect Immun 65: 3713-3718, 1997.

3. Wright AC, Powell JL, Kaper JB and Morris JG Jr: Identification of a group 1-like capsular polysaccharide operon for Vibrio vulnificus. Infect Immun 69: 6893-6901, 2001.

4. Simpson LM and Oliver JD: Siderophore production by Vibrio vulnificus. Infect Immun 41: 644-649, 1983.

5. Gray LD and Kreger AS: Purification and characterization of an extracellular cytolysin produced by Vibrio vulnificus. Infect Immun 48: 67-72, 1985.

6. Gulig PA, Bourdage KL and Starks AM: Molecular Pathogenesis of Vibrio vulnificus. J Microbiol 43: 118-131, 2005.

7. Lee JH, Kim MW, Kim BS, Kim SM, Lee BC, Kim TS and Choi SH: Identification and characterization of the Vibrio vulnificus rtxA essential for cytotoxicity in vitro and virulence in mice. J Microbiol 45: 146-152, 2007.

8. Lee BC, Lee JH, Kim MW, Kim BS, Oh MH, Kim KS, Kim TS and Choi SH: Vibrio vulnificus rtxE is important for virulence, and its expression is induced by exposure to host cells. Infect Immun 76: 1509-1517, 2008.

9. Lee BC, Choi SH and Kim TS: Vibrio vulnificus RTX toxin plays an important role in the apoptotic death of human intestinal epithelial cells exposed to Vibrio vulnificus. Microbes Infect 10: 1504-1513, 2008.

10. Espat NJ, Auffenberg T, Abouhamze A, Baumhofer J, Moldawer LL and Howard RJ: A role for tumour necrosis factor- $\alpha$ in the increased mortality associated with Vibrio vulnificus infection in the presence of hepatic dysfunction. Ann Surg 223: 428-433, 1996.

11. Shin SH, Shin DH, Ryu PY, Chung SS and Rhee JH: Proinflammatory cytokine profile in Vibrio vulnificus septicemic patients' sera. FEMS Immunol Med Microbiol 33: 133-138, 2002.

12. Suh JK and Matthew HW: Application of chitosan-based polysaccharide biomaterials in cartilage tissue engineering: a review. Biomaterials 21: 2589-2598, 2000.

13. Peluso G, Petillo O and Ranieri M: Chitosan-mediated stimulation of macrophage function. Biomaterials 15: 1215-1220, 1994.

14. Usami Y, Okamoto Y, Minami S, Matsuhashi A, Kumazawa NH, Tanioka S and Shigemasa Y: Chitin and chitosan induce migration of bovine polymorphonuclear cells. J Vet Med Sci 56: 761-762, 1994.

15. Usami Y, Okamoto Y, Minami S, Matsuhashi A, Kumazawa NH, Tanioka S and Shigemasa Y: Migration of canine neutrophils to chitin and chitosan. J Vet Med Sci 56: 1215-1216, 1994.

16. Usami Y, Okamoto Y, Takayama T, Shigemasa Y and Minami S: Chitin and chitosan stimulate canine polymorphonuclear cells to release leukotriene B4 and prostaglandin E2. J Biomed Mater Res 42: 517-522, 1998.

17. Kim TH, Jin H, Kim HW, Cho MH and Cho CS: Mannosylated chitosan nanoparticle-based cytokine gene therapy suppressed cancer growth in BALB/c mice bearing CT-26 carcinoma cells. Mol Cancer Ther 5: 1723-1732, 2006.

18. Qi L and Xu Z: In vivo antitumor activity of chitosan nanoparticles. Bioorg Med Chem Lett 16: 4243-4245, 2006.

19. Maeda Y and Kimura Y: Antitumor effects of various lowmolecular-weight chitosans are due to increased natural killer activity of intestinal intraepithelial lymphocytes in sarcoma 180bearing mice. J Nutr 134: 945-950, 2004.

20. Schlievert PM: Chitosan malate inhibits growth and exotoxin production of toxic shock syndrome-inducing Staphylococcus aureus strain and group A streptococci. Antimicrob Agents Chemother 51: 3056-3062, 2007. 
21. Tré-Hardy M, Vanderbist F, Traore H and Devleeschouwer MJ: In vitro activity of antibiotic combinations against Pseudomonas aeruginosa biofilm and planktonic cultures. Int J Antimicrob Agents 31: 329-336, 2008.

22. Seyfarth F, Schliemann S, Elsner P and Hipler UC: Antifungal effect of high- and low-molecular-weight chitosan hydrochloride, carboxymethyl chitosan, chitosan oligosaccharide and $\mathrm{N}$-acetylD-glucosamine against Candida albicans, Candida krusei and Candida glabrata. Int J Pharm 353: 139-148, 2008.

23. Hossain S, Rahman A, Kabir Y, Shams AA, Afros F and Hashimoto M: Effects of shrimp (Macrobracium rosenbergii)derived chitosan on plasma lipid profile and liver lipid peroxide levels in normo- and hypercholesterolaemic rats. Clin Exp Pharmacol Physiol 34: 170-176, 2007.

24. Geremias R, Pedrosa RC, Locatelli C, de Fávere VT, Coury-Pedrosa R and Laranjeira MC: Lipid lowering activity of hydrosoluble chitosan and association with Aloe vera $L$. and Brassica olearaceae L. Phytother Res 20: 288-293, 2006.

25. Sumiyoshi M and Kimura Y: Low molecular weight chitosan inhibits obesity induced by feeding a high-fat diet long-term in mice. J Pharm Pharmacol 58: 201-207, 2006.

26. Wang P, Jiang X, Jiang Y, Hu X, Mou H, Li M and Guan H: In vitro antioxidative activities of three marine oligosaccharides. Nat Prod Res 21: 646-654, 2007.

27. Zhong Z, Ji X, Xing R, Liu S, Guo Z, Chen X and Li P: The preparation and antioxidant activity of the sulfanilamide derivatives of chitosan and chitosan sulfates. Bioorg Med Chem 15: 3775-3782, 2007.

28. Cho MH, No HK and Prinyawiwatkul W: Chitosan treatments affect growth and selected quality of sunflower sprouts. J Food Sci 73: S70-S77, 2008.
29. Moon JS, Kim HK, Koo HC, Joo YS, Nam HM, Park YH and Kang MI: The antibacterial and immunostimulative effect of chitosan-oligosaccharides against infection by Staphylococcus aureus isolated from bovine mastitis. Appl Microbiol Biotechnol 75: 989-998, 2007.

30. Kim SK and Rajapakse N: Enzymatic production and biological activities of chitosan oligosaccharides (COS): A review. Carbohydr Polym 62: 357-368, 2005.

31. Jeon YJ and Kim SK: Chitosan derivatives killed bacteria by disrupting the outer and inner membrane. J Agric Food Chem 54: 6629-6633, 2006.

32. Yoon HJ, Moon ME, Park HS, Im SY and Kim YH: Chitosan oligosaccharide (COS) inhibits LPS-induced inflammatory effects in RAW 264.7 macrophage cells. Biochem Biophys Res Commun 358: 954-959, 2007.

33. Linkous DA and Oliver JD: Pathogenesis of Vibrio vulnificus. FEMS Microbiol Lett 174: 207-214, 1999.

34. Strom MS and Paranjpye RN: Epidemiology and pathogenesis of Vibrio vulnificus. Microbes Infect 2: 177-188, 2000.

35. Tharanathan RN and Kittur FS: Chitin--the undisputed biomolecule of great potential. Crit Rev Food Sci Nutr 43: 61-87, 2003.

36. Vishu Kumar AB, Varadaraj MC, Gowda LR and Tharanathan RN: Characterization of chito-oligosaccharides prepared by chitosanolysis with the aid of papain and Pronase, and their bactericidal action against Bacillus cereus and Escherichia coli. Biochem J 391(Pt 2): 167-175, 2005.

37. Kim WH, Goo SY, Shin MH, Chun SJ, Lee H, Lee KH and Park SJ: Vibrio vulnificus-induced death of Jurkat T-cells requires activation of p38 mitogen-activated protein kinase by NADPH oxidase-derived reactive oxygen species. Cell Immunol 253: 81-91, 2008. 